\title{
Mechanism of surface dehydration of anatase $\left(\mathrm{TiO}_{2}\right)$
}

\author{
A. Córdoba and J. J. Luque \\ Departamento de Termología, Facultad de Física, Universidad de Sevilla, Sevilla, Spain \\ (Received 18 September 1984; revised manuscript received 5 February 1985)
}

\begin{abstract}
A comparative study of the dehydration of the (001) and (111) surfaces of $\mathrm{TiO}_{2}$ (anatase) has been made starting from a master equation and introducing suitable transition probabilities. From the experimental data considered, relative to sample "Degussa P25" of Munuera and co-workers, it is concluded that the (111) face is the most likely to be exposed. The detailed mechanism of dehydration is analyzed and an estimate of the values of activation energies and frequency factors is presented. This analysis makes it clear that the surface migration of protons plays an important role during the dehydration process. Also the influence on the degree of surface hydration, the dehydration rate, and the numbers of certain ionic groups on the surface exerted by the temperature rate in a linear heating program and by initial surface coverage are discussed.
\end{abstract}

\section{INTRODUCTION}

The surface of titanium dioxide has been the subject of much research. ${ }^{1}$ Interest in these studies has been increased because the photoactivity of the $\mathrm{TiO}_{2}$ surface can play an important role in solar energy conversion. ${ }^{2-7}$ Specifically, a number of these studies have focused on analyzing the hydroxyl groups on the surface and the defects created by removal of these hydroxyl groups. Titanium dioxide in its anatase form exposes preferably the (001) (Ref. 8) and (111) (Ref. 9) cleavage planes, both of which are very different, and both faces have been considered in interpreting experimental data. Removal of water from a hydroxylated surface involves two adjacent hydroxyl groups, and moreover, the surface sites are, in general, of various types. In two previous papers, we developed a method of studying the kinetics of adsorption and desorption with and without surface diffusion, both where the process takes place by paired adatoms ${ }^{10}$ and where the surface is heterogeneous. ${ }^{11}$. Applying this method we can design a model of the surface dehydration of anatase in order to delve deeper into the knowledge of the basic mechanisms governing the process and to follow the evolution of certain ionic groups on the surface. Thus, we start from a master equation with transition probabilities suitably chosen and we obtain the kinetic equations governing this phenomenon. By comparing our results with experimental data it is possible to perform an estimate of the values of a number of parameters characterizing the basic mechanisms of dehydration. The outline of this paper is as follows: In Sec. II we describe the (111) face of anatase and the mechanism of dehydration and formulate the kinetic model; in Sec. III we estimate the values of the parameters in the kinetic equations, solve these equations and analyze the results obtained, and, finally, in Sec. IV we consider the (001) face of anatase and a comparison between both (111) and (001) faces is made.

\section{KINETIC MODEL OF THE (111) FACE}

First, let us consider the (111) face of anatase. According to Munuera et al. ${ }^{9}$ the (111) face of anatase may be represented by a triangular lattice consisting of stepped parallel rows, called (0), (1), (2), and (3) [the (0) row is the most external and the (3) one is the most internal], periodically repeated four by four (see Fig. 1). The hydrated surface can retain water in molecular form, which is easy to remove, and as hydroxyl groups. In an idealized model one may assume that all adsorbed water is as hydroxyl groups, which form a monolayer with $15.2 \mathrm{OH}^{-}$groups per $100 \AA^{2}$ for a fully hydroxylated surface. Indeed this $\mathrm{OH}^{-}$density is high, and the fully hydroxylated surface is likely unstable. The situation of the (0) and (3) rows seems less stable than that of the (1) and (2) rows, because an analysis of the coordination of the surface ions makes it evident that the (0) and (3) hydroxyl groups have uncompensated charges $-\frac{1}{3}$ and $+\frac{1}{3}$, respectively, while in the (1) and (2) rows ions with uncompensated charges $+\frac{1}{3}$ and $-\frac{1}{3}$ alternate. Thus in a fully hydroxylated surface, (0) and (3) rows have a net uncompensated charge, but (1) and (2) are electrically neutral.

It is assumed that the surface is fully hydroxylated at the initial time. When the temperature is raised, adjacent hydroxyl ions combine to form water molecules, which are then desorbed. The facility to remove two adjacent hydroxyls is associated with the facility to exchange a proton between each adjacent hydroxyl group. When two adjacent hydroxyl groups are removed, an oxide ion and a vacant site are created on the lattice (they form an "asymmetrical dimer"). Removal of hydroxyl groups can
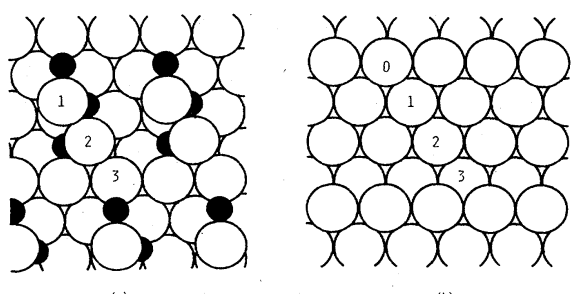

FIG. 1. Ideal (111) cleavage plane of anatase: (a) dehydroxylated surface, where white and black circles are oxide and titanium ions, respectively; (b) fully hydroxylated surface, where white circles are hydroxyl groups. 
take place as follows, where the elemental mechanisms from greater to lesser facility to remove hydroxyl groups are enumerated.

(i) Removal of a (0) hydroxyl group together with a (3) hydroxyl group, leaving an oxide ion in the (3) row and a vacant site in the $(0)$ one (solid line in Fig. 2).

(ii) Removal of two hydroxyl groups, both located in the (1) row, both in the (2) row, or one in the (1) row and the other in the (2) row. Then an oxide ion and a vacant site are created and are randomly located on the pair of sites previously occupied by the removed hydroxyls (broken line in Fig. 2).

(ii) Removal of a (0) hydroxyl group together with a (1) hydroxyl group, leaving a vacant site in the $(0)$ row and an oxide ion in the (1) row. Removal of a (2) hydroxyl group together with a (3) hydroxyl group, creating a vacant site in the (2) row and an oxide ion in the (3) (dotted line in Fig. 2).

(iv) Removal of two adjacent hydroxyl groups both in the (0) row or both in the (3) row is not possible (dotted and dashed lines in Fig. 2).

It should be noted that, starting from a fully hydroxy-

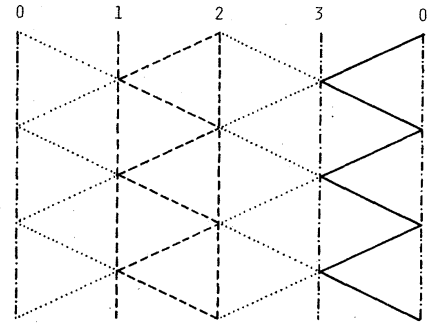

FIG. 2. Lattice associated with (111) cleavage plane of anatase and scheme of dehydroxylation mechanism (see text).

lated surface, oxide ions in the $(0)$ row and vacant sites in the (3) row are not created, because a surface oxide ion must be shared by two cations $\mathrm{Ti}^{4+}$, and an oxide ion in the $(0)$ row would be coordinated with only a cation as a double ligand, which is very unlikely. On the other hand, a vacancy in the (3) row would create an internal cation (i.e., a nonsurface cation) with incomplete coordination and an surface oxide ion coordinate with only a cation.

Each elemental process can be summarized as follows:

$2 \mathrm{OH}^{-}($surface $) \rightarrow \mathrm{O}^{2-}($ surface $)+$ vacant site $($ surface $)+\mathrm{H}_{2} \mathrm{O}$ (vapor $)$

and, in general, they are characterized by different reaction rates.

In addition to these removal processes, diffusion of protons on the lattice must also be considered. If $i$ and $j$ are adjacent sites occupied by a hydroxyl group and an oxide ion, respectively, the exchange between both of a proton can be represented as

$\mathrm{OH}^{-}(i$ site $)+\mathrm{O}^{2-}(j$ site $) \rightarrow \mathrm{O}^{2-}(i$ site $)+\mathrm{OH}^{-}(j$ site $)$

If diffusion is not operative, some hydroxyl groups become isolated, i.e., do not have another adjacent hydroxyl, and then their removal is impossible. However, when there is diffusion, if some hydroxyl group becomes isolated, but has an adjacent oxide ion, it is possible that during the dehydration process this oxide ion will accept a proton of another neighboring hydroxyl; then a hydroxylhydroxyl pair can be created and a water molecule can be removed from the surface according to the mechanisms described above. Also it is possible that an isolated hydroxyl group may be left fully enclosed by vacant sites and remain indefinitely on the lattice, but this is very unlikely and the number of these hydroxyl groups is negligible. Finally it is observed that readsorption is not operative, because, for normal experimental conditions, desorbed water is immediately evacuated.

According to the mechanisms described above, a kinetic model for dehydration of the (111) face of anatase is designed.

At an intermediate stage of dehydration, the surface can be considered as a system composed of hydroxyl groups $(H)$, oxide ions $(O)$, and vacant sites $(V)$. A variable $s_{i j}$ for each lattice site is associated which takes the values

$$
s_{i j}=\left\{\begin{array}{l}
-1 \text { for a vacant site } \\
0 \text { for a site occupied by a hydroxyl group } \\
+1 \text { for a site occupied by an oxide ion }
\end{array}\right.
$$

i.e., the system is similar to a two-dimensional spin-1 system.

The guideline of Refs. 10 and 11 is followed and the master equation is formulated. The elemental process changing a state of the system, according to the mechanism described above, can be summarized as follows:

\begin{tabular}{cc} 
Removal & Diffusion \\
\hline$H_{3} H_{0} \rightarrow O_{3} V_{0}$ & $O_{1} H_{1} \rightarrow H_{1} O_{1}$ \\
$H_{1} H_{1} \rightarrow \begin{cases}O_{1} V_{1} & H_{1} O_{1} \rightarrow O_{1} H_{1} \\
V_{1} O_{1} & O_{1} H_{2} \rightarrow H_{1} O_{2}\end{cases}$ \\
$H_{1} H_{2} \rightarrow \begin{cases}O_{1} V_{2} & H_{1} O_{2} \rightarrow O_{1} H_{2} \\
V_{1} O_{2} & O_{2} H_{2} \rightarrow H_{2} O_{2}\end{cases}$ \\
$H_{2} H_{2} \rightarrow \begin{cases}O_{2} V_{2} & H_{2} O_{2} \rightarrow O_{2} H_{2} \\
V_{2} O_{2} & H_{3} O_{3} \rightarrow O_{3} H_{3}\end{cases}$ \\
$H_{0} H_{1} \rightarrow V_{0} O_{1}$ & $O_{3} H_{3} \rightarrow H_{3} O_{3}$ \\
$H_{2} H_{3} \rightarrow V_{2} O_{3}$ & $H_{2} O_{3} \rightarrow O_{2} H_{3}$
\end{tabular}

where the subscript indicates the row where $H, O$, or $V$ is located.

We have not considered the cases $\mathrm{H}_{0} \mathrm{O}_{1} \rightarrow \mathrm{O}_{0} \mathrm{H}_{1}$, $\mathrm{O}_{3} \mathrm{H}_{0} \rightarrow \mathrm{H}_{3} \mathrm{O}_{0}, \mathrm{H}_{0} \mathrm{O}_{0} \rightarrow \mathrm{O}_{0} \mathrm{H}_{0}$, or $\mathrm{O}_{0} \mathrm{H}_{0} \rightarrow \mathrm{H}_{0} \mathrm{O}_{0}$ for the diffusion process, because we assume that oxide ions are never created in the most external row, as we have previously indicated. 
The reaction rate for each removal elemental process is chosen in the Arrhenius form:

$$
\begin{aligned}
& f_{i j}^{1}=A_{i j}^{1} \exp \left(-E_{i j}^{1} / R T\right), \\
& f_{i j}^{2}=A_{i j}^{2} \exp \left(-E_{i j}^{2} / R T\right),
\end{aligned}
$$

where $i$ and $j$ subscripts denote the rows where the removed $H$ 's are placed ( $j=i$ or $j=i+1$; for the latter case, $j=0$ if $i=3$ ), and the 1 and 2 superscripts distinguish between the pairs $O-V$ (superscript 1) and $V-O$ (superscript 2) which can be created from a pair $H$ - $H$. In Eqs. (1), $A_{i j}^{1}$ and $A_{i j}^{2}$ are frequency factors, $E_{i j}^{1}$ and $E_{i j}^{2}$ are molal activation energies, $R$ is the universal gas constant, and $T$ is the temperature.

Likewise the diffusion process rate can be written:

$$
\begin{aligned}
& g_{i j}^{1}=A_{d i j}^{1} \exp \left(-E_{d i j}^{1} / R T\right), \\
& g_{i j}^{2}=A_{d i j}^{2} \exp \left(-E_{d i j}^{2} / R T\right),
\end{aligned}
$$

Here $A_{d i j}^{1}$ and $A_{d i j}^{2}$ can be considered as the product of the frequency factor of the dissociation $\mathrm{OH}^{-} \rightarrow \mathrm{O}^{2-}+\mathrm{H}^{+}$by the reciprocal of a proper time of the "physical" diffusion. As will be discussed later, this proper diffusion time can be several hours, therefore $A_{d i j}^{1}$ and $A_{d i j}^{2}$ are much less than if they were only frequency factors of dissociation processes. On the other hand, diffusion energy is less than desorption energy, and the former is usually much less than the latter in chemisorption processes. Thus it is reasonable to assume that $E_{d i j}^{1}$ and $E_{d i j}^{2}$ are much less than $E_{i j}^{1}$ and $E_{i j}^{2}$, respectively.

By introducing the expression of transition probabilities in the master equation, the basic equation for this model is obtained. By starting from this equation, the kinetic equation can be calculated.

The process will be described in terms of $n_{i}^{l}$ and $n_{i j}^{l m}$, where $n_{i}^{l}$ denotes the fraction of lattices sites in the row $i$ $(i=0,1,2,3)$ in a state $l(l=V, H, O)$, and $n_{i j}^{l m}$ denotes the fraction of pairs in adjacent sites. The $n_{i}^{H}$ also will be written $\theta_{i}$, the total hydration degree being $\theta=\sum_{i} \theta_{i}$.

Taking into account the relationships among the various $n_{i}^{l}$ and $n_{i j}^{l m}$ and choosing a set of independent variables, we obtain a set of coupled equations where the rates $d n_{i}^{l} / d t$ and $d n_{i j}^{l m} / d t$ are expressed in terms of the quantities $n_{i}^{l}, n_{i j}^{l m}, \mathscr{A}_{i j k}^{l m n}$, and $\mathscr{B}_{i j k}^{l m n}$, where $\mathscr{A}_{i j k}^{l m n}$ and $\mathscr{B}_{i j k}^{l m n}$ denote the fractions of clusters shown in Fig. 3, i.e., the time evolution of the singlet and doublet fractions, $n_{i}^{l}$ and $n_{i j}^{l m}$, depend also on the triplet fractions $\mathscr{A}_{i j k}^{l m n}$ and $\mathscr{B}_{i j k}^{l m}$.

Altogether, thirty-six equations are obtained (eight for $d n_{i}^{l} / d t$ and twenty-eight for $\left.d n_{i j}^{l m} / d t\right)$. They form an open hierarchy, because $\mathscr{A}_{i j k}^{l m n}$ and $\mathscr{B}_{i j k}^{l m n}$ are involved. This is typical of the applied method and it is necessary to make an additional assumption to close this hierarchy. Making an extension of the closure approximations applied in Refs. 10 and 11, we assume

$$
\begin{aligned}
& \mathscr{A}_{i j k}^{l m n}=n_{i j k} \frac{\left(n_{i j}^{l m} / n_{i j}\right)\left(n_{j k}^{m n} / n_{j k}\right)}{\left(n_{j}^{m} / n_{j}\right)}, \\
& \mathscr{B}_{i j k}^{l m n}=n_{i j k} \frac{n_{i j}^{l m}}{n_{i j}} \frac{n_{j k}^{m n}}{\sum_{\alpha} n_{j k}^{m \alpha}} .
\end{aligned}
$$
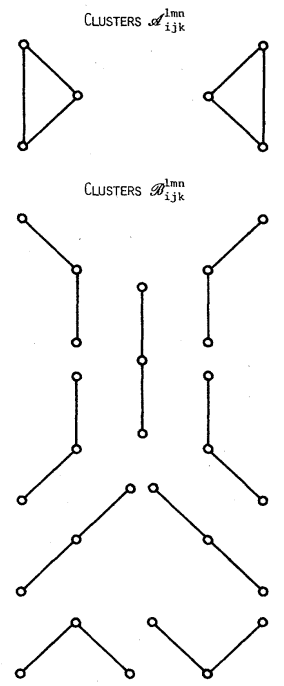

FIG. 3. Clusters $\mathscr{A}_{i j k}^{l m n}$ and $B_{i j k}^{l m n}$.

Thus we obtain a closed system, where the time rate of the densities of singlets $n_{i}^{l}$ and doublets $n_{i j}^{l m}$ are nonlinear functions of these densities. Numerical methods must be applied to solve these kinetic equations.

Now the value of the parameters involved in the kinetic equations must be fixed. These parameters are the preexponential factors and the activation energies in Eqs. (1) and (2). Because there are no clear criteria to fix their values, certain ranges which can be expected from general aspects of the problem are considered and next an estimate of these parameters within the previous ranges is made by fitting experimental data of the thermogravimetric analysis. Because the experimental data show wide scatter for different samples, the data of a specific sample are chosen and this fact must be taken into account in considering the obtained values.

According to the diffusion and removal mechanisms (i)-(iv), previously described, and considering that elemental process with equal probability to occur have the same values for their characteristic parameters, we assume

$$
\begin{aligned}
A_{30}^{1} & =A_{1}, \\
A_{11}^{1} & =A_{11}^{2}=A_{12}^{1}=A_{12}^{2}=A_{22}^{1}=A_{22}^{2}=A_{2}, \\
A_{01}^{2} & =A_{23}^{2}=A_{3}, \\
A_{d 11}^{1} & =A_{d 11}^{2}=A_{d 12}^{1}=A_{d 12}^{2}=A_{d 22}^{1}=A_{d 22}^{2} \\
& =A_{d 33}^{1}=A_{d 33}^{2}=A_{d 23}^{1}=A_{d 23}^{2}=A_{d} .
\end{aligned}
$$

This assumption reduces the number of preexponential factors to four; three factors for removal, $A_{1}, A_{2}$, and $A_{3}$, and one factor for diffusion, $A_{d}$. With regard to energy, we consider only one activation energy for removal, $E$, and another for diffusion, $E_{d}$.

First, we analyze the values of the energies. For these processes a typical ratio between desorption activation energy and diffusion activation energy can be taken as $10 .^{12}$ Thus we assume $E_{d}=0.1 E$. In general, $E$ is a function of 
the hydration degree $\theta$, and $E$ increases as $\theta$ decreases. Although there are not direct calorimetric measurements, the variation range of $E$ must be within the range $\simeq 40$ $\mathrm{kJ} \mathrm{mol}^{-1}$ (vaporization heat of water) to $\simeq 100 \mathrm{~kJ} \mathrm{~mol}^{-1}$ (heat of surface hydration estimated from the heat of immersion in water of anatase $\left.{ }^{13}\right)$, and we assume a linear relationship between $E$ and $\theta(E=c-a \theta, c>0, a>0)$ because it is the easiest relationship and because calorimetric measurements have shown a linear relationship between desorption activation energy and hydration degree for surface dehydration of other oxides. ${ }^{14}$

On the other hand, typical values of frequency factors for dimolecular processes controlled by a surface reaction are within the range $10^{4}-10^{8} \mathrm{~min}^{-1} \cdot{ }^{12,15}$ For removal of pairs 3-0, where hydroxyl groups have a greater freedom to vibrate, the highest value, i.e., $A_{1} \simeq 10^{8} \mathrm{~min}^{-1}$ can be expected, while $A_{3}$ takes the lowest value, i.e., $A_{3} \simeq 10^{4}$ $\min ^{-1}$, since hydroxyl groups corresponding to this process are the hardest to remove and it is possible that they have lost some rotational degrees of freedom. Thus, we assume $A_{1}=10^{8} \mathrm{~min}^{-1}$ and $A_{3}=10^{4} \mathrm{~min}^{-1}$. The value $A_{2}$ will be taken halfway between $A_{1}$ and $A_{3}$, nearly $10^{6}$ $\min ^{-1}$. Finally we must fix the value of $A_{d}$, the product of the frequency factor of the reaction which leaves a proton by the reciprocal time characterizing diffusion. The frequency factor is nearly within the above-mentioned range of $10^{4}-10^{8} \mathrm{~min}^{-1}$, but the diffusion process is very slow, according to experimental evidence. Thus the value of $A_{d}$ must be much lower than $10^{4} \mathrm{~min}^{-1}$. Summarizing, we choose $A_{1}=10^{8} \min ^{-1}, A_{3}=10^{4} \mathrm{~min}^{-1}$, $E=c-a \theta, E_{d}=0.1 E$, and we must determine $A_{2}\left(\sim 10^{6}\right.$ $\left.\min ^{-1}\right), \quad A_{d}\left(\ll 10^{4} \min ^{-1}\right), \quad$ and $a$ and $c \quad(40$ $\left.\mathrm{kJ} \mathrm{mol}^{-1}<c-a \theta<100 \mathrm{~kJ} \mathrm{~mol}^{-1}\right)$. With this object we perform a comparison between results of our model and experimental data of thermogravimetric analysis of a sample of anatase "Degussa P25" studied by Munuera and co-workers. ${ }^{16,17}$ In looking for a qualitative agreement between results of our model and experimental data, we have made an estimate of the values of the parameters.

\section{RESULTS AND DISCUSSION}

Sample "Degussa P25" of Munuera and co-workers has been submitted to a linear heating program $\left(T=T_{0}+b t\right)$ with a temperature rate $b=7.5{ }^{\circ} \mathrm{Cmin}^{-1}$. We assume that at the initial time the surface is fully hydroxylated $(\theta=1)$ and there is no molecular water on the surface. This assumption is perhaps rather extreme, but it is not exactly possible to establish the initial state of the system from the available experimental data.

A comparison of the experimental data with the computed curve $\theta-T$, using $E=89-30 \theta \mathrm{kJ} \mathrm{mol}^{-1}, A_{2}=1.9$ $\times 10^{6} \mathrm{~min}^{-1}$, and $A_{d}=5 \times 10^{-1} \mathrm{~min}^{-1}$, is shown in Fig. 4. The agreement of the numerical results and the experimental data is good and the values of the parameters estimated are within the expected ranges.

On the other hand, a comparison between the calculated curve $-d \theta / d T$ versus $T$ and that obtained by Munuera and co-workers ${ }^{16,17}$ by differential thermogravimetric analysis shows a qualitative agreement between calculations and experimental data. The derivatographic curve
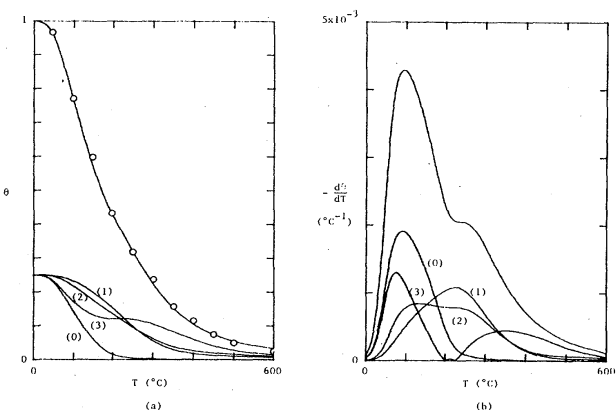

FIG. 4. (a) Degree of hydroxylation versus temperature, where (0), (1), (2), and (3) denote contribution to total coverage by (0), (1), (2), and (3) rows, respectively. White circles are experimental points (Ref. 17). (b) Dexydroxylation rate versus temperature.

obtained by Munuera and co-workers exhibits a maximum at $\simeq 100^{\circ} \mathrm{C}$, a "shoulder" at $\simeq 215^{\circ} \mathrm{C}$, and a long tail above $300^{\circ} \mathrm{C}$; the total curve is then resolved in three curves, corresponding to three types of adsorbed water, giving peaks at $\simeq 100^{\circ} \mathrm{C}, 215^{\circ} \mathrm{C}$, and $325^{\circ} \mathrm{C}$. Our calculated curve is similar to the experimental curve, although the shoulder is slightly shifted towards the right-hand side and the decomposition in curves corresponding to different types of adsorbed water is more complex than the analysis of Munuera and co-workers [see Fig. 4(b)]. For the value of $A_{2}$ which differs a little from the indicated one, the shoulder becomes a small sharp maximum, as experimental results for other samples show (for example, the sample called $c$ in Ref. 9).

By analyzing Figs. 4 and 5 one can clarify the dehydration process in detailed form. The important role played by proton diffusion becomes apparent. If migration of protons is not operative, hydroxyl groups (3) together with hydroxyl groups $(0)$ would be removed almost completely at relatively low temperatures and hydroxyl groups (1) and (2) would be removed at higher temperatures, both dehydration stages partially overlapping, leaving a remainder of about $5 \%\left(0.76\right.$ hydroxyl groups per $\left.100 \AA^{2}\right)$ on the surface. However, due to diffusion, which is more effective at high temperatures but also operates at low temperatures, the dehydration mechanism is more complex. Indeed at the beginning, removal of hydroxyl group (0)-hydroxyl group (3) pairs starts, and vacant sites on
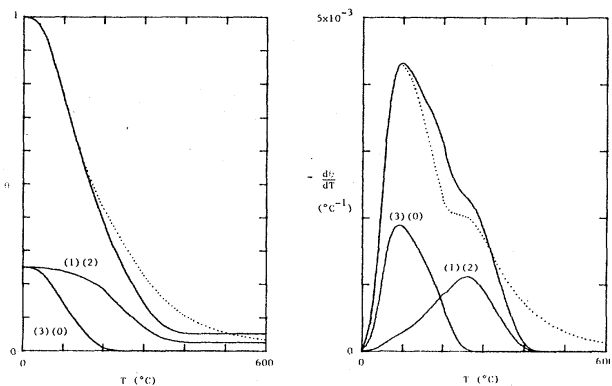

FIG. 5. Comparison of results obtained with (dotted line) and without (solid line) proton diffusion. For the case without diffusion, evolution of rows (1) and (2) and rows (3) and (0) coincide. 
the (0) row and oxide ions on the (3) row are created. As the (3) row has an uncompensated charge $+\frac{1}{3}$ by hydroxyl group and $-\frac{2}{3}$ by oxide ion and the (2) row has hydroxyl groups with uncompensated charge $+\frac{1}{3}$ and $-\frac{1}{3}$, alternatively, there is a tendency that the (2) row (neutral on the whole) give protons to the (3) row (which increases its uncompensated negative charge as the process goes forward), in order to diminish the surface charge heterogeneity. This fact yields that $-d \theta_{0} / d T$ is greater than $-d \theta_{3} / d T$, since hydroxyls removed from the (3) row are in part counterbalanced by diffusion of protons from the (2) row. On the other hand, $-d \theta_{2} / d T$ is greater than $-d \theta_{1} / d T$, not because (2) hydroxyl groups are removed more quickly than (3) hydroxyl groups, but because those give a proton to the (3) row and become oxide ions. A comparison of the results obtained with and without proton mobility makes clear that $\theta_{1}$ and $\theta_{0}$ have similar behavior for both cases, while $\theta_{3}$ and $\theta_{2}$ do not (see Figs. 4 and 5). Therefore the dehydroxylation process takes place essentially as follows: There is a first stage between $0^{\circ} \mathrm{C}$ and $\simeq 200^{\circ} \mathrm{C}$ where practically all the (0) hydroxyl groups are entirely removed, with a maximum for the desorption rate at $T \simeq 100^{\circ} \mathrm{C}$, and the (3) hydroxyl-group removal is in part counterbalanced by proton diffusion from (2) row to (3) row, holding a remainder $\theta_{3} \simeq 0.12$. There is a second stage, partially overlapped with the first one, between $50^{\circ} \mathrm{C}$ and $400^{\circ} \mathrm{C}$, with a maximum desorption rate at $T \simeq 225^{\circ} \mathrm{C}$, where removal takes place in the (1) and (2) rows. And finally, in a third stage, overlapped in part with the second one, with a maximum in the curve $-d \theta_{3} / d T$ versus $T$ at $T \simeq 350^{\circ} \mathrm{C}$ and a long tail above this temperature, protons migrate from the (3) row to the (2) and (1) rows, where hydroxyls are slowly removed.

Next we analyze another aspect of the problem. We have assumed a linear heating program, in such a way that temperature increases as time does, and accordingly the hydration degree decreases. But if at a medium stage of the process the temperature is fixed and the evolution of the system is observed, one can note that the dehydroxylation process proceeds, although in a very much slower manner. Figure 6 shows the behavior of $\theta$ versus $t$, when the temperature is fixed at $250^{\circ} \mathrm{C}, 350^{\circ} \mathrm{C}$, and $450^{\circ} \mathrm{C}$, respectively, and evolution of system is followed

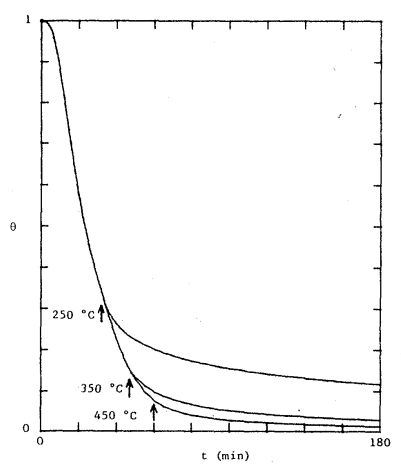

FIG. 6. Time evolution of the hydroxylation degree when the heating program is cut off for a given temperature. Arrows indicate the time and the temperature of the cutoff. for three hours. As $E_{d}$ is low, the diffusion rate $A_{d} \exp \left(-E_{d} / R T\right)$ depends smoothly on $T$, and for high temperature this rate is nearly equal to the constant $A_{d}$; thus $A_{d}$ is the parameter which essentially determines the diffusion mechanism. This fact indicates that at not too high temperature, when hydroxyl-group removal is partially performed and diffusion is operative, if one waits sufficient time (in general, many hours or even several days), it is possible to observe that the full surface dehydroxylation is actually achieved. There is experimental evidence of this fact. It follows that the knowledge of the state of the surface of a sample obtained for specific conditions can lose validity in the course of time.

Another interesting question is the evolution of certain surface ionic groups, specifically the pairs. Obviously the number of hydroxyl-hydroxyl pairs decreases as the process advances (see Fig. 7), and its evolution is consistent with the above description of the dehydration process. The number of oxide-hydroxyl pairs, which makes proton diffusion possible, starts from zero, passes through a maximum and finally decays again to zero. One must note (see Fig. 7) that the highest maximum corresponds to $n_{23}^{\mathrm{HO}}$ for the initial stage [this fact facilitates proton diffusion from (2) row to (3) row], and to $n_{23}^{O H}$ for the final stage [thus facilitating the reverse diffusion process from (3) row to (2) row].

In order to analyze surface reactivity, the oxide-oxide and vacancy-vacancy pairs can be considered. Two adjacent oxides ions create a local excess of negative charge, while two adjacent vacant sites create a local excess of positive charge. From Fig. 7, by comparison with the number of pairs corresponding to an ideal dehydroxylated surface (see Fig. 1), it is apparent that $n_{23}^{O O}$ and $n_{12}^{H H}$, at $T=600^{\circ} \mathrm{C}$, differ slightly from the ideal values 0.25 and 0.125 , respectively, while $n_{33}^{O O} \simeq 0.08$ (ideal value 0.25 ), $n_{22}^{00} \simeq 0.05$ (ideal value zero), and $n_{11}^{00} \simeq 0.06$ (ideal value
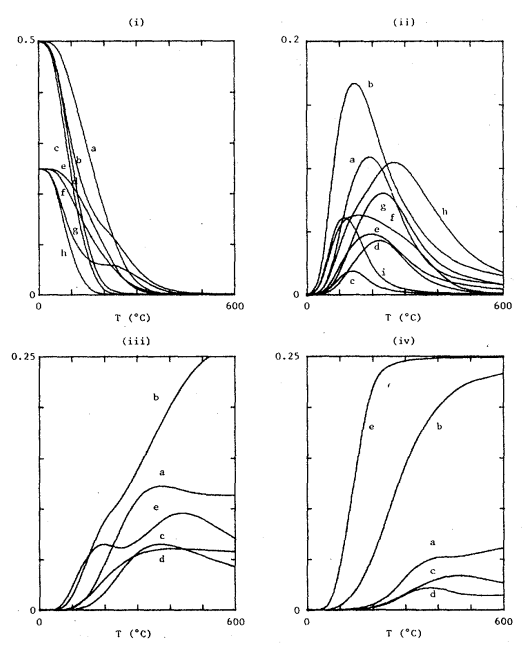

FIG. 7. Evolution with temperature of number of pairs. (i) Hydroxyl-hydroxyl pairs: $a, n_{12}^{H H} ; b, n_{23}^{H H} ; c, n_{30}^{H H} ; d, n_{01}^{H H} ; e$, $n_{11}^{H H} ; f, n_{22}^{H H} ; g, n_{33}^{H H} ; h, n_{0 O}^{H H}$. (ii) Hydroxyl-oxide and oxidehydroxyl pairs: $a, n_{12}^{H O} ; b, n_{23}^{H O} ; c, n_{01}^{H O} ; d, n_{11}^{H O} ; e, n_{22}^{H O} ; f, n_{33}^{H O}$; $g, n_{12}^{O H}, h, n_{23}^{O H} ; i, n_{30}^{O H}$. (iii) Oxide-oxide pairs: $a, n_{12}^{O O} ; b, n_{23}^{O O}$; $c, n_{11}^{O O} ; d, n_{22}^{O O} ; e, n_{33}^{O 0}$. (iv) Vacancy-vacancy pairs: $a, n_{12}^{V V} ; b$, $n_{01}^{V V} ; c, n_{11}^{V V} ; d, n_{22}^{V V} ; e, n_{00}^{V V}$. 
zero). Thus, the (3) row holds a number of oxide-oxide pairs much lower than the expected number for an ideal dehydroxylated surface, i.e., it has a minor excess of negative charge, while the (1) and (2) rows have a greater excess of negative charge than the ideal dehydroxilated surface. Therefore, (1) and (2) rows must exhibit a greater reactivity than that expected for an ideal surface, due to their tendency to gain protons to diminish their excess of charge.

Evolution of vacancy-vacancy pairs is also shown in Fig. 7. By comparison of the results at $T=550^{\circ} \mathrm{C}$ with the ideal dehydroxylated surface, a low value of $n_{12}^{V V}$ is obtained, this fact indicating that there are local excesses of negative charge in the (1) and (2) rows, as has already been mentioned.

If some hydroxyl groups are left on the surface when the heating is cut off, later diffusion of protons and removal of water can modify the number of pairs. But if $\theta$ is small (a few percent) when the heating is.cut off, these modifications are negligible and the above analysis remains valid.

Another parameter to be considered is the temperature rate $b$. Calculations have been made for $b=16{ }^{\circ} \mathrm{C} \mathrm{min}-1$ and the results have been compared with those previously obtained for $b=7.5^{\circ} \mathrm{C} \mathrm{min}^{-1}$ (see Figs. 8 and 9).

In Fig. 8 the curve $\theta$ versus $T$ is shown for both values of $b$; the curve for $b=16^{\circ} \mathrm{C} \mathrm{min}^{-1}$ is shifted towards the right-hand side with regard to the curve for $b=7.5$ ${ }^{\circ} \mathrm{C} \min ^{-1}$ (i.e., for a given temperature $\theta$ increases as $b$ does), but both curves are qualitatively similar. Analogous behavior is exhibited by the curve $d \theta / d T$ versus $T$. Obviously, if one plots the results versus $t$ instead of $T$, one can note that the process takes place much more quickly for $b=16{ }^{\circ} \mathrm{Cmin}^{-1}$ than for $b=7.5^{\circ} \mathrm{C} \mathrm{min}^{-1}$.

Concerning the evolution of ionic groups with the dehydration degree and the temperature rate, the total number of hydroxyl-hydroxyl, hydroxyl-oxide and oxide-hydroxyl, and oxide-oxide pairs for both values of $b$ are shown in Fig. 9. One can note the influence of $b$ is negligible or minimal. Perhaps one might expect that a quick heating process produces a more disordered dehydroxylation process than a slow one, but our analysis makes it apparent that temperature rate has very little influence on the number of ionic groups for a given $\theta$. Thus for the surface of a given sample of anatase submitted to different heating
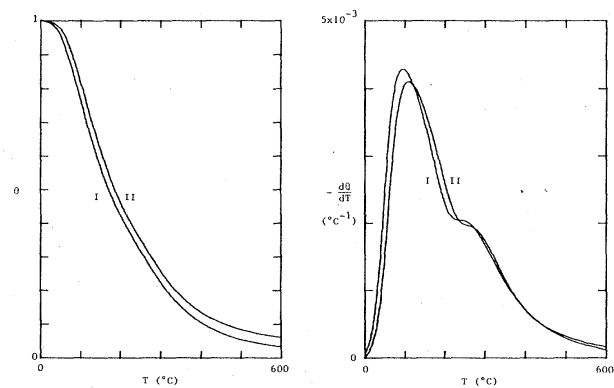

FIG. 8. Comparison of the results obtained for different temperature rates: I, $b=7.5^{\circ} \mathrm{Cmin}^{-1}$; II, $b=16^{\circ} \mathrm{C} \mathrm{min}^{-1}$.
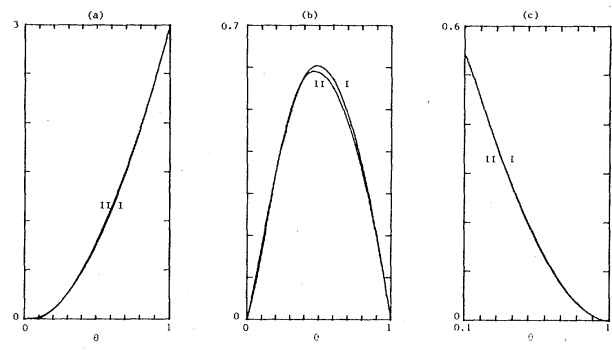

FIG. 9. Evolution with hydroxylation degree of the total number of (a) hydroxyl-hydroxyl, (b) hydroxyl-oxide and oxidehydroxyl, and (c)-oxide-oxide pairs for different temperature rates: I, $b=7.5^{\circ} \mathrm{C} \mathrm{min}^{-1}$; II, $b=16^{\circ} \mathrm{C} \mathrm{min}^{-1}$.

programs, we may conclude that its chemical and spectroscopical properties depend essentially on $\theta$, but not on the temperature rate.

Finally, we shall analyze the influence of initial conditions. Thus far we have been considering a fully hydroxylated surface as a starting point, but this condition is probably ideal. In Fig. 10 we compare two cases, curves $b$ and $c$, for an incomplete initial coverage, with the case of full initial hydroxylation (curve $a$ ). For curve $b$, $\theta_{\text {initial }}=0.6$ and initial $n_{i}^{k}$ and $n_{i j}^{k l}$ take the values for the curve $a$ for $\theta=0.6$. The curve $\theta$ versus $T$ shows that at the beginning removal of hydroxyl groups is not operative until a sufficiently high temperature is attained and then the process continues almost the same as the $a$ case. The curve $-d \theta / d T$ versus $T$ exhibits two maxima, the first maxima being much less sharp than corresponding one for the $a$ case, and it is shifted towards the right-hand side. For the $c$ case, $\theta_{\text {initial }}=0.7$ and $n_{i}{ }^{k}$ and $n_{i j}^{k l}$ take values randomly, compatible with the value of $\theta$. The initial configuration randomly chosen is very unstable because at the beginning the dehydroxylation process is very abrupt and the value of $\theta$ suddenly becomes nearly 0.3 . Then the process stops until a sufficiently high temperature is attained and henceforth the evolution of the system is similar to that observed for the $a$ and $b$ curves. Ignoring the sudden initial dehydroxylation, the curve $-d \theta / d T$ versus $T$ exhibits two maxima, the maximum at high temperatures being higher than the maximum at low temperatures.

When molecular water and hydroxyl ions coexist on the surface, a superposition of the curves corresponding to re-
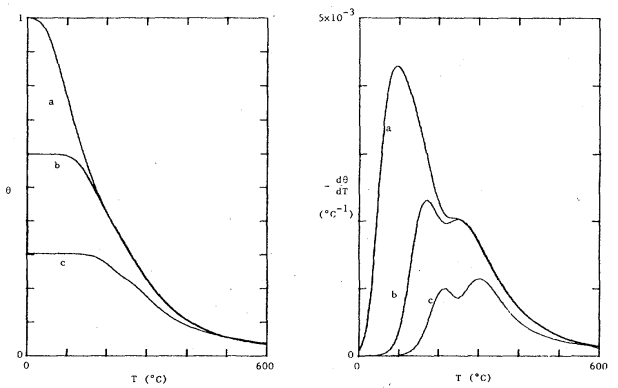

FIG. 10. Dehydroxylation for different initial conditions: $a$, $\theta_{\text {initial }}=1 ; b, \theta_{\text {initial }}=0.6$ and initial distribution of pairs equal to that resulting in $a$ for $\theta=0.6 ; c, \theta_{\text {initial }}=0.7$ and random initial distribution of pairs. 
moval of both types of water is necessary, providing that both processes do not interact. Because experimental data often involve both processes, a sample submitted to different processes of dehydration and further rehydration can produce different curves of $\theta$ versus $T$ for the same value of $\theta$, since initial conditions may be different, and thus it is very important to characterize accurately the initial state of the surface, which is hardly ever done by experimentalists.

From the above analysis we conclude that our model acceptably explains the experimental data of dehydroxylation of certain samples of anatase and provides an effective method of characterizing the resulting dehydroxylated surface.

\section{DEHYDROXYLATION OF THE (001) FACE}

The model of the (001) face of anatase fully dry and fully hydroxylated is shown in Fig. 11. The most external and the most internal surface hydyroxyl groups have an excess of uncompensated charge $-\frac{1}{3}$ and $+\frac{1}{3}$, respective1y. Schematically the fully hydroxylated (001) face of anatase can be represented by a rectangular lattice, where each elemental rectangle is $1.89 \AA \times 3.78 \AA$ (14.2 sites per $100 \AA^{2}$ ) and where particles of two different types, called 0 and 1 , are allocated as Fig. 12 shows. There one can see that two particles of the same type are at least $3.78 \AA$ apart, while two adjacent particles of different types are only $1.89 \AA$ apart. Thus, due to the distance between hydroxyl groups and to the sign of their excess of charge, removal of hydroxyl groups is probably achieved by $0-1$ pairs, allocated in the same row. In our study, since hydroxyl groups of different chains are never jointly removed, we can consider the surface as a set of independent linear chains, as indicated in Fig. 12, and we shall deal with a one-dimensional system.

At a medium stage of dehydration there are 0 - and 1hydroxyl groups on the surface and therefore the surface is heterogeneous. Results from, for example, infrared spectroscopy will indicate this fact by exhibiting two different bands. However, with regard to results of thermogravimetric analysis, the behavior of the surface will be similar to that of a homogeneous surface, because removal always involves a 0 - and 1 -hydroxyl group jointly. Therefore, we expect the curve $-d \theta / d T$ versus $T$ to exhibit only a maximum.

Removal of two adjacent hydroxyl groups creates a va-

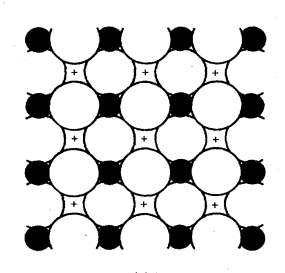

FIG. 11. Ideal (001) cleavage plane of anatase: (a) dehydroxylated surface; (b) fully hydroxylated surface. Dotted, white, and black circles are, respectively, hydroxyl groups, oxide ions, and titanium ions, and + denotes more internal oxide ions.

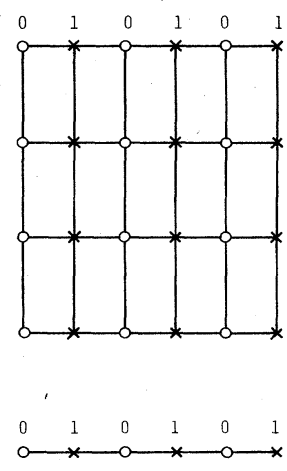

FIG. 12. Lattice associated with the (001) cleavage plane of anatase and linear chain where the dehydroxylation of the (001) face takes place.

cant site and an oxide ion on the chain, and we assume that both are randomly allocated on the two sites previously occupied by the removed hydroxyl groups. Proton diffusion is also permitted as

$$
\mathrm{OH}^{-}(j)+\mathrm{O}^{2-}(k) \rightarrow \mathrm{O}^{2-}(j)+\mathrm{OH}^{-}(k),
$$

where $j$ and $k$ denote adjacent sites. It is possible that one hydroxyl group has two adjacent vacant sites (vacancyhydroxyl-vacancy triplet) and becomes unremovable, but its number would be very low.

As for the (111) face, we can obtain the kinetic equations (there are eight equations-four for singlets and four for doublets), which involve the quantities

$$
f_{i}=A_{i} \exp \left(-E_{i} / R T\right) \quad(i=1,2)
$$

corresponding to the elemental process of removal

$\mathrm{OH}^{-}+\mathrm{OH}^{-} \rightarrow\left\{\begin{array}{ll}\mathrm{O}^{2-}+\text { vacancy } & \text { for } i=1 \\ \text { vacancy }+\mathrm{O}^{2-} & \text { for } i=2\end{array}\right\}+\mathrm{H}_{2} \mathrm{O}$ (vapor)

and

$$
g_{i}=A_{d i} \exp \left(-E_{d i} / R T\right) \quad(i=1,2)
$$

corresponding to the diffusion mechanism

$$
\begin{aligned}
& \mathrm{OH}^{-}+\mathrm{O}^{2-} \rightarrow \mathrm{O}^{2-}+\mathrm{OH}^{-} \text {for } i=1 \\
& \mathrm{O}^{2-}+\mathrm{OH}^{-} \rightarrow \mathrm{OH}^{-}+\mathrm{O}^{2-} \text { for } i=2
\end{aligned}
$$
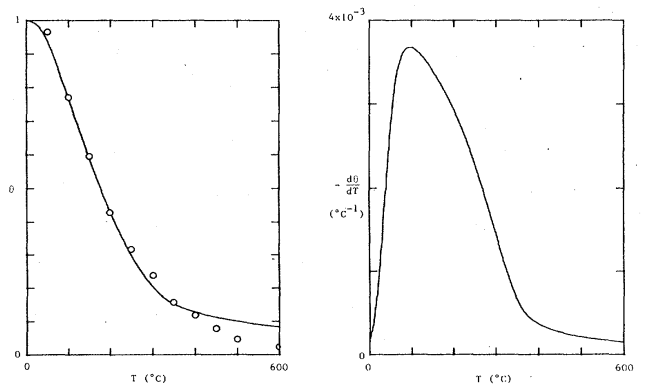

FIG. 13. Dehydroxylation of the (001) surface of anatase with $A_{1}=A_{2}=10^{7} \mathrm{~min}^{-1}, E_{1}=E_{2}=95-40 \theta \mathrm{kJ} \mathrm{mol}^{-1}, A_{d 1}$ $=A_{d 2}=0.7 \mathrm{~min}^{-1}$, and $E_{d 1}=E_{d 2}=0.1 E_{1}$. White circles are experimental points (Ref. 17). 
When we have tried to fit the experimental data of the sample "Degussa P25," using this model, we have found that it is possible to fit the range of low temperatures or the range of high temperatures, but it is not possible to fit both simultaneously. Also the curve $-d \theta / d T$ versus $T$ exhibits one maximum and one tail, but it is not possible to obtain one shoulder or one maximum intermediate between these. This fact can be seen in Fig. 13, where one of the tried fits is shown, with

$$
\begin{aligned}
& A_{1}=A_{2}=10^{7} \mathrm{~min}^{-1}, \\
& E_{1}=E_{2}=95-40 \theta \mathrm{kJ} \mathrm{mol}^{-1},
\end{aligned}
$$

$$
\begin{aligned}
& A_{d 1}=A_{d 2}=0.7 \mathrm{~min}^{-1}, \\
& E_{d 1}=E_{d 2}=0.1 E_{1} .
\end{aligned}
$$

This result agrees with the hypothesis of Munuera and co-workers that the preferably exposed face for this type of sample is the (111) face. Due to the samples being polycrystalline in general, it is probable that both (111) and (001) faces coexist, the (111) one prevailing. This mixed situation can be treated by superimposing the results of Sec. III and Sec. IV, suitably weighted.

\section{ACKNOWLEDGMENT}

We would like to thank Professor G. Munuera for helpful discussions.
${ }^{1}$ See, for example, the review by G. D. Parfitt, Progress in Surface and Membrane Science (Academic, New York, 1976), Vol. 11, p. 181

${ }^{2}$ A. Fujishima and K. Honda, Nature (London) 238, 37 (1972).

${ }^{3}$ M. S. Wrighton, D. S. Ginley, P. T. Workzanski, and A. B. Ellis, Proc. Nat. Acad. Sci. USA 72, 1918 (1975).

${ }^{4}$ J. G. Mavroides, D. S. Tchernev, J. A. Kafalas, and D. F. Kolesar, Mater. Res. Bull 10, 1023 (1975).

${ }^{5}$ P. Clechet, F. Juillet, and R. Oliver, J. Chim. Phys. 4, 396 (1976).

${ }^{6}$ P. A. Kohl, S. N. Frank, and A. J. Bard, J. Electrochem. Soc. 129, 226 (1977).

${ }^{7}$ K. Nakatani and H. Tsubomura, Bull. Chem. Soc. Jpn. 60, 183 (1977).

${ }^{8}$ M. Primet, J. Basset, M. V. Mathieu, and M. Prettre, J. Phys. Chem. 74, 2868 (1970).

${ }^{9}$ G. Munuera, F. Moreno, and F. Gonzalez, in Reactivity of
Solids, edited by J. S. Anderson, M. W. Roberts, and F. S. Stone (Chapman and Hall, London, 1972), p. 681.

10J. J. Luque and A. Córdoba, J. Chem. Phys. 76, 6393 (1982).

${ }^{11}$ A. Córdoba and J. J. Luque, Phys. Rev. B 26, 4028 (1982); 28, 7353 (1983).

${ }^{12}$ P. Malet, Tesina de Licenciatura, University of Seville, 1978 (unpublished).

${ }^{13}$ T. Morimoto, M. Nagao, and T. Omori, Bull. Chem. Soc. Jpn. 42, 943 (1969).

${ }^{14} \mathrm{~F}$. Rouquerol, S. Regnier, and J. Rouquerol, Therm. Anal. 1, 313 (1974).

${ }^{15}$ H. F. Cordes, J. Phys. Chem. 72, 2185 (1968).

${ }^{16} \mathrm{G}$. Munuera, V. Rives-Arnau, and A. Saucedo, J. Chem. Soc. Faraday Trans. 75, 736 (1979).

${ }^{17}$ A. Saucedo, Tesina de Licenciatura, University of Seville, 1977 (unpublished). 